\title{
Interactive comment on "Where there is smoke there is mercury: Assessing boreal forest fire mercury emissions using aircraft and highlighting uncertainties associated with upscaling emissions estimates” by David S. McLagan et al.
}

\section{Anonymous Referee \#3}

Received and published: 1 February 2021

The authors present aircraft measurements of GEM, CO, $\mathrm{CO} 2, \mathrm{CH} 4$, and NMHCs in the plume of a forest fire in Saskatchewan. From these data they derive the emission ratios and calculate GEM emissions by three different methods. In addition, they calculate GEM flux using the screen flown downwind of the fire. The results of GEM emissions calculated by different methods are compared and their uncertainties discussed. 
The purpose of the extensive discussion of the GEM enhancements is not clear. In addition, it will strongly depend on the meteorology which is omitted from the discussion. Without consideration of the windspeed (dilution) and the distance to the fire, the comparison with measurements published by other authors does not make much sense.

Section 2.1: Measurement of wind speed and direction onboard aircraft is not easy. The reader would like to know how these parameters were measured and with which uncertainties. This information is needed to assess the uncertainty of fluxes calculated by TERRA.

Sections 2.2 and 2.3: What are the estimated uncertainties of the individual GEM, $\mathrm{CO}, \mathrm{CO} 2$, and $\mathrm{CH} 4$ measurements. These uncertainties are needed for assessment of the quality of ERs: e.g. the poorer quality of $\mathrm{GEM} / \mathrm{CH} 4$ ratio could be caused by higher uncertainty of $\mathrm{CH} 4$ measurements? They are also needed for the orthogonal correlations (Cantrell, ACP, 8, 5477-5487, 2008).

Line 233: I presume the GEM background is given as an average of 2 min measurements. What was the number of the GEM measurements used in this average?

Lines 237-241: The consideration of only GEM enhancements $>125 \%$ is probably not justified for several reasons: a) It is arbitrary - why not $115 \%$ ? b) The selection of $>125 \%$ GEM data may show only a part of the plume which may not be representative of the whole plume. c) With increasing distance to the fire, the section of plume with $>125 \%$ would decrease relatively to the whole plume which again poses the question of representativity. d) The authors state that the data below $125 \%$ the enhancement are "too variable and too uncertain" to be considered. The concern about the uncertainty should not be the problem if the authors used orthogonal regression with uncertainties of both GEM and $X$. The variability should also be no problem: the more points the smaller R is significant. Reference to Yokelson et al. (2013) is not quite appropriate for the situation here, i.e. with measurements up to $100 \mathrm{~km}$ distance from the fire with

Printer-friendly version

Discussion paper
Interactive

comment 
nearly constant background mixing ratios.

In addition, the limitation to GEM enhancements $>125 \%$ even seems to be unnecessary: the ERs in figure S4.1 calculated from all data and in Table 1 calculated with only GEM enhancements $>125 \%$ are probably the same in statistical terms, i.e. cannot be distinguished taking into account the ERs uncertainties and the number of measurements. An additional table of ERs from Table 1 and ERs from figure S4.1 could be used to illustrate the necessity of the $>125 \%$ threshold or its absence.

Paragraph lines 245-248: What type of regression was used: LSQF or orthogonal one? The usual LSQF should not be used to calculate ERs!!! The last sentence is difficult to understand because any type of regression automatically adjusts for the backgrounds - it is just a shift in the coordinate system. Probably $\mathrm{CO}, \mathrm{CO} 2, \mathrm{CH} 4$, and $\mathrm{NMHC}$ measurements were converted to 2 min averages synchronized with 2 min GEM for correlations? If so, it should be mentioned.

Paragraph lines 249-252: How was the integration made? Only for the enhancements $>125 \%$ ?

Paragraph lines 305-327: More information is needed in the description of TERRA calculation? How were GEM measurements $(2 \mathrm{~min})$ interpolated? The treatment of the layer above the highest transection and the inversion layer has to be mentioned too. Some of this information is provided in Section 3.4 but the reader would expect it here.

Lines 348 and 349: How was the correlation made: orthogonal? 2min data? What are the regression lines?

Paragraph lines 459-464: The conversion of 2 min GEM measurements into $0.5 \mathrm{~Hz}$ data using the GEM/CO ratio was probably made mainly for the TERRA calculations. If so, it should be mentioned.

Printer-friendly version

Paragraph 465-480: The text here is highly speculative because the estimations are 
strongly dependent on the meteorology which is not mentioned. In addition, it does not contribute much to the purpose of the paper.

Table 1: The comparison of maximal measured GEM enhancements is strongly dependent on their temporal resolution, as shown in this work, and on the meteorological parameters (especially wind speed in combination with the distance to the fire, i.e. dilution). Without taking all these factors into account, the comparison does not make much sense and as such should be deleted from the table, and also from the text.

Table 2: "Uncertainty" should be used instead of "error" here and throughout the text. The calculation of uncertainties and the terms used in the equation 7 should be given either in the manuscript or in the supporting information.

Fig. 2b: I wonder about 2s GEM data derived from $0.5 \mathrm{~Hz}$ CO data using the GEM/CO ratio. What is this conversion good for? I find its presentation misleading because it pretends much higher density of GEM data than available. The $0.5 \mathrm{~Hz} \mathrm{CO}, \mathrm{CO} 2$, and $\mathrm{CH} 4$ measurements should be converted to the 2 min GEM time stamp, at least for the regressions.

Fig. 3: Which type of regression was used? The usual one (LSQF) or one which considers the $X$ and the $Y$ uncertainties? What was the number of correlated points: $R$ without the number of points does not say anything about the significance of the regression. Dtto the figure S4.1.

Fig. 5: "Distance" in the name of $x$ axis may be easily mixed up with the distance to fire.

Interactive comment on Atmos. Chem. Phys. Discuss., https://doi.org/10.5194/acp-2020-1119, 2020. 